\title{
Exploring the transition into an asymmetric planetary nabulae
}

\author{
Mónica W. Blanco Cárdenas ${ }^{1}$, Roberto Vázquez ${ }^{1}$, \\ Hans Ulrich Kaeufl ${ }^{2}$, Marco A. Gómez-Muñoz ${ }^{1}$, Pedro F. Guillén ${ }^{1}$, \\ Martín A. Guerrero ${ }^{3}$ and Luis F. Miranda ${ }^{3}$ \\ ${ }^{1}$ Instituto de Astronomía, Universidad Nacional Autónoma de México, \\ Km 103 Carretera Tijuana-Ensenada 22860, Ensenada, Mexico \\ email: mwblanco@astrosen.unam.mx \\ ${ }^{2}$ European southern Observatory, \\ Karl Schwarchild-Str 2 85748, Garching bei Muenchen, Germany \\ ${ }^{3}$ Instituto de Astofísica de Andalucía, \\ Glorieta de la Astronomía S/N 18008, Granada, Spain
}

\begin{abstract}
We present studies using different observational techniques, along different frequencies, aiming to resolve and investigate jets, outflows, as well as compact and innermost regions of asymmetric planetary nebulae $(\mathrm{PNe})$ and objects in transition to $\mathrm{PN}$. All the information gathered allow us to explore the kinematics and other important properties of the structures that play a crucial role in the shaping of complex PNe morphologies, in particular, we explore the role of disks/tori as collimating engine of extreme axisymmetric PNe.
\end{abstract}

Keywords. Stars, circumstellar mater, outflows.

\section{The complex sculpting of an asymmetric planetary nebula}

Several planetary nebulae (PNe) are asymmetric and high velocity jets and outflows are common features in these evolved objects. Furthermore, during the last years, steady and/or slowly rotating disks have been discovered in the cores of extreme axisymmetric PN. Infrared observations provide the opportunity to investigate the innermost dusty regions that usually are related with torii and disks. In this work, we present proto-PNe and young PNe with at least one observational proof of torus or disk, and we explore these structures using other techniques: spectro-astrometry (SA) using CRIRES-VLT (Blanco Cárdenas et al. 2014 ) and analysis of its SED using available photometry of several missions from the visible to radio frequencies (2MASS, MSX, WISE, IRAS, AKARI, etc.) and the SED building and analysis tool IRIS (cxc.cfa.harvard.edu/iris/v2.1).

\section{Overview and preliminary results}

Proto-PN AFGL 915. This source (a.k.a The Red Rectangle) has a well-known rotating disk of few hundreds of AU (Bujarrabal et al. (2013)) and references there in) surrounded by a steady disk of silicates (Waters et al. (1998)). Blanco Cárdenas et al. (2014) detected a smaller spectral signature of this rotating disk (few tens of AU, $\sim 100$ mas) using the SA technique. As for the SED, three components fit well: a $7000 \mathrm{~K}$ Blackbody (BB), a rotating disk in mid-IR wavelengths, and a $170 \mathrm{~K} \mathrm{BB}$ for the larger wavelengths.

IRAS 18454+0001: an extremely young PN? Given its compact size $(\sim 1 ")$ and the lack of an optical counterpart this source must be a bipolar PN in the making. 

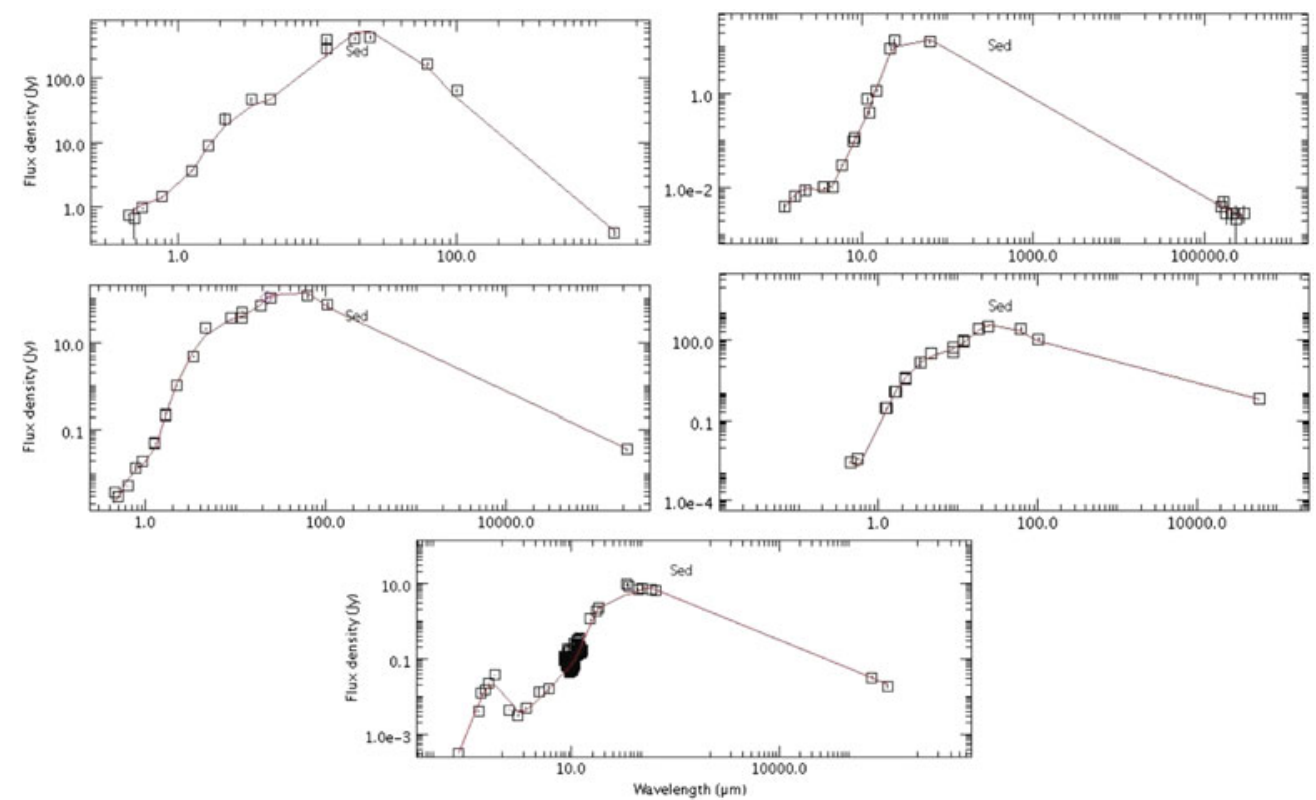

Figure 1. SEDs of the sample. From left to right and up to bottom: AFGL 915,

IRAS 18454+0001, M 2-9, Mz 3, and M 1-16.

Results outstanding the equatorial estructure clearly dominated by the $\lambda 8.6 \mu \mathrm{m} \mathrm{PAH}$ filter of VISIR-VLT (Blanco Cárdenas et al. 2013 ), whereas the spitzer spectrum shows the presence of carbon-rich material (mid-IR PAH features) and confirms the presence of ionised material ([NeII] $\lambda 12.8 \mu \mathrm{m})$. According to the SED fitting, a very hot star $(83,000 \mathrm{~K})$ and a disk beyond the mid-IR (larger dust grains) are needed, among other components of warmer material in the IR.

Young PNe: M 2-9, Mz 3 and M 1-16. Silicate disks were found in the central regions of M 2-9 and Mz 3 (Chesneau et al. 2007 and Lykou et al. 2011). On the other hand, CO $(J=2-1)$ observations confirms the presence of two concentric rings in M 2-9 (CastroCarrizo et al. 2012) and a torus in M 1-16 (Huggins et al. 2000). These three sources have fast collimated outflows (up to $\sim 300 \mathrm{kms}^{-1}$ ). Our CRIRES spectra does not show any evidence of disks in M 2-9 and Mz 3, only the origin of the bipolar lobes of M 2-9 is likely detected. The SEDs of M 2-9 and Mz 3 are alike. However, for M 2-9 two BB of $96,000 \mathrm{~K}$ and $3,200 \mathrm{~K}$ fit the optical data, whereas only one $\mathrm{BB}$ of $30,000 \mathrm{~K}$ fits the $\mathrm{SED}$ of $\mathrm{Mz} 3$. A rotating disk in the mid-IR and components around $100 \mathrm{~K}$ fit the longer wavelengths. M 1-16 has a prominent optical component fitted by $32,000 \mathrm{~K} \mathrm{BB}$. A rotating disk fits well the data, nevertheless, this disk may have a hotter component in the near IR. Once again, colder BBs are needed to fit the data beyond mid-IR wavelengths.

\section{References}

Blanco Cárdenas, M., Kaeulf, H. U., Guerrero, M. A. et al. 2014, AछA, 566, A133

Blanco Cárdenas, Guerrero, M. A., Ramos-Larios, G et al. 2013, A\&A, 551, A64

Bujarrabal, V., Castro-Carrizo, A., Alcolea, J., et al. 2013, A $\mathscr{E} A$, 557, L11

Castro-Carrizo, A., Neri, R., Bujarrabal, V. et al. 2012, A\&A, 545, A1

Chesneau, O., Lykou, B., Balick, B. et al. 2007, A\&A, 473, L29

Huggins, P. J., Forveille, T., Bachiller, R., \& Cox, P. 2000, ApJ, 544, 889

Lykou, B., Chesneau, O., Zijlstra, A. A. et al. 2011, A\& A, 527, A105

Waters, L. B. F.. M., Waelkens, C., van Winckel, H. et al. 1998, Nature, 67, 4679 